\title{
A Novel Exactly Theoretical Solvable of Bound States of the Dirac-Kratzer-Fues Problem with Spin and Pseudo-Spin Symmetry
}

\author{
Abdelmadjid Maireche \\ Laboratory of Physics and Material Chemistry, Physics department, Sciences Faculty, \\ University of M'sila-M'sila Algeria. \\ abmaireche@gmail.com
}

Keywords: The Kratzer-Fues potential, Noncommutative space, Star product, Bopp's shift method and Dirac equation.

\begin{abstract}
New exact bound state solutions of the deformed radial upper and lower components of Dirac equation and corresponding Hermitian anisotropic Hamiltonian operator are studied for the modified Kratzer-Fues potential (m.k.f.) potential by using Bopp's shift method instead to solving deformed Dirac equation with star product. The corrections of energy eigenvalues are obtained by applying standard perturbation theory for interactions in one-electron atoms. Moreover, the obtained corrections of energies are depended on two infinitesimal parameters $(\Theta, \chi)$, which induced by position-position noncommutativity, in addition to the discreet nonrelativistic atomic quantum numbers: $(j=\tilde{l} \pm 1 / 1, s= \pm 1 / 2, \tilde{l}$ and $\widetilde{m})$ and we have also shown that, the usual relativistic states in ordinary three dimensional spaces are canceled and has been replaced by new degenerated $2(2 \tilde{l}+1)$ sub-states in the extended quantum symmetries (NC: 3D-RS).
\end{abstract}

\section{Introduction}

In relativistic quantum mechanics, one of the interesting problems is to obtain exact solutions of the Klein-Gordon equation (spin zero particle) and Dirac equation (spin $1 / 2$ particle) at high energy, much interest in providing analytic solutions to the relativistic equations in many fields of Physics and Chemistry for different central and non central potentials [1-16]. The quantum structure based to the ordinary canonical commutations relations in both Schrödinger and Heisenberg (the operators are depended on time) pictures, respectively (Natural units $c=\hbar=1$ are employed throughout this paper):

$$
\left\lfloor x_{i}, p_{j}\right\rfloor=i \delta_{i j} \quad \text { and }\left\lfloor x_{i}, x_{j}\right\rfloor=\left\lfloor p_{i}, p_{j}\right\rfloor=0
$$

and

$$
\left\lfloor x_{i}(t), p_{j}(t)\right\rfloor=i \delta_{i j} \text { and }\left\lfloor x_{i}(t), x_{j}(t)\right\rfloor=\left\lfloor p_{i}(t), p_{j}(t)\right\rfloor=0
$$

where the two operators $\left(x_{i}(t), p_{i}(t)\right)$ in Heisenberg picture are related to the corresponding two operators $\left(x_{i}, p_{i}\right)$ from the two projections relations:

$$
\begin{aligned}
& x_{i}(t)=\exp \left(i \hat{H}\left(t-t_{0}\right)\right) x_{i} \exp \left(-i \hat{H}\left(t-t_{0}\right)\right) \\
& p_{i}(t)=\exp \left(i \hat{H}\left(t-t_{0}\right)\right) p_{i} \exp \left(-i \hat{H}\left(t-t_{0}\right)\right)
\end{aligned}
$$

here $\hat{H}$ denote to the ordinary quantum Hamiltonian operator. In addition, for spin $1 / 2$ particles described by the Dirac equation, experiment tells us that must satisfy Fermi Dirac statistics obey the restriction of Pauli, which imply to gives the only non-null equal-time anti-commutator for field operators as follows:

$$
\left\{\Psi_{\alpha}(t, r), \bar{\Psi}_{\beta}\left(t, \mathrm{r}^{\prime}\right)\right\}=i \gamma_{\alpha \beta}^{0} \delta^{3}\left(\mathrm{r}-\mathrm{r}^{\prime}\right)
$$


with $\bar{\Psi}_{\beta}\left(t, \mathrm{r}^{\prime}\right)=\Psi^{+}{ }_{\beta}\left(t, \mathrm{r}^{\prime}\right) \gamma^{0}$. It is important to notice that, the noncommutativity idea was introduced firstly by H. Snyder and A. Connes in 1946 and 1986, respectively [17,18]. Very recently, much considerable effort has been expanded on the solutions of Schrödinger, Dirac and Klein-Gordon equations to noncommutative quantum mechanics [19-59], the new quantum structure of noncommutative space based on the following noncommutative canonical commutations relations in both Schrödinger and Heisenberg pictures, respectively, as follows:

$$
\begin{aligned}
& {\left[\hat{x}_{i}^{*}, \hat{p}_{j}\right]=i \delta_{i j},\left[\hat{x}_{i}^{*}, \hat{x}_{j}\right]=i \theta_{i j} \quad \text { and }\left[\hat{p}_{i}^{*}, \hat{p}_{j}\right]=0} \\
& {\left[\hat{x}_{i}(t)^{*}, \hat{p}_{j}(t)\right]=i \delta_{i j},\left[\hat{x}_{i}(t)^{*}, \hat{x}_{j}(t)\right]=i \theta_{i j} \text { and }\left[\hat{p}_{i}(t)^{*}, \hat{p}_{j}(t)\right]=0}
\end{aligned}
$$

The above new quantum structure allow us to realize the non-commutative Moyal spaces, the two new operators $\left(\hat{x}_{i}(t), \hat{p}_{i}(t)\right)$ in Heisenberg picture are related to the corresponding operators $\left(\hat{x}_{i}, \hat{p}_{i}\right)$ in Schrödinger picture from the two projections relations:

$$
\begin{aligned}
& \hat{x}_{i}(t)=\exp \left(i \hat{H}_{n c}\left(t-t_{0}\right)\right) * \hat{x}_{i} * \exp \left(-i \hat{H}_{n c}\left(t-t_{0}\right)\right) \\
& \hat{p}_{i}(t)=\exp \left(i \hat{H}_{n c}\left(t-t_{0}\right)\right) * \hat{p}_{i} * \exp \left(-i \hat{H}_{n c}\left(t-t_{0}\right)\right)
\end{aligned}
$$

Here $\hat{H}_{n c}$ denote to the new quantum Hamiltonian operator in (NC: 3D-RS) symmetries. The very small parameters $\theta^{\mu \nu}$ (compared to the energy) are elements of antisymmetric real matrix and $(*)$ denote to the new star product, which is generalized between two arbitrary functions $f(x)$ and $g(x)$ to $(f * g)(x)$ instead of the usual product $(f g)(x)$ in ordinary three dimensional spaces:

$$
(f * g)(x) \equiv \exp \left(\frac { i } { 2 } \theta ^ { \mu v } \partial _ { \mu } ^ { x } \partial _ { \nu } ^ { x } ( f g ) ( x ) \equiv \left(f g-\left.\frac{i}{2} \theta^{\mu v} \partial_{\mu}^{x} f \partial_{\nu}^{x} g\right|_{\left(x^{\mu}=x^{v^{\nu}}\right)}+O\left(\theta^{2}\right)\right.\right.
$$

the first term on the right side gives the ordinary product, the term $\left(-\frac{i}{2} \theta^{\mu v} \partial_{\mu}^{x} f(x) \partial_{\nu}^{x} g(x)\right)$ is induced by (space-space) noncommutativity properties and $O\left(\theta^{2}\right)$ stands for the second and higher order terms of $\theta$, a Bopp's shift method can be used, instead of solving any deformed quantum systems by using directly star product procedure:

$$
\left\lfloor\hat{x}_{i}, \hat{x}_{j}\right\rfloor=i \theta_{i j} \text { and }\left\lfloor\hat{p}_{i}, \hat{p}_{j}\right\rfloor=0
$$

The new three-generalized coordinates $\left(\hat{x}=\hat{x}_{1}, \hat{y}=\hat{x}_{2}, \hat{z}=\hat{x}_{3}\right)$ are depended with corresponding threeusual generalized positions $(x, y, z)$ and momentum coordinates $\left(p_{x}, p_{y}, p_{z}\right)$ by the following relations, as follows:

$$
\begin{aligned}
& \hat{x}=x-\frac{\theta_{12}}{2} p_{y}-\frac{\theta_{13}}{2} p_{z} \\
& \hat{y}=y-\frac{\theta_{21}}{2} p_{x}-\frac{\theta_{23}}{2} p_{z} \\
& \hat{z}=z-\frac{\theta_{31}}{2} p_{x}-\frac{\theta_{32}}{2} p_{y}
\end{aligned}
$$

The non-vanish -commutators in (NC-3D: RS) can be determined as follows:

$$
\begin{aligned}
& {\left[\hat{x}, \hat{p}_{x}\right]=\left[\hat{y}, \hat{p}_{y}\right\rfloor=\left[\hat{z}, \hat{p}_{z}\right]=i,} \\
& {[\hat{x}, \hat{y}]=i \theta_{12},[\hat{x}, \hat{z}]=i \theta_{13},[\hat{y}, \hat{z}]=i \theta_{23}}
\end{aligned}
$$


which allow us to getting the operator $\hat{r}^{2}$ on noncommutative three dimensional spaces as follows:

$$
\hat{r}^{2}=r^{2}-\overrightarrow{\mathbf{L}} \vec{\Theta}
$$

where the coupling $\mathbf{L} \Theta$ is given by $\left(\Theta_{i j}=\frac{\theta_{i j}}{2}\right)$ :

$$
\mathbf{L} \Theta \equiv L_{x} \Theta_{12}+L_{y} \Theta_{23}+L_{z} \Theta_{13}
$$

with:

$$
L_{x}=y p_{z}-z p_{y}, L_{y}=\mathrm{zp}_{\mathrm{x}}-\mathrm{xp}_{\mathrm{z}} \text { and } L_{z}=x p_{y}-y p_{x}
$$

Furthermore, the non-null equal-time anti-commutator for fermionic field operators in noncommutative spaces can be expressed in the following postulate relations:

$$
\begin{aligned}
& \left\{\hat{\Psi}_{\alpha}(t, r), \hat{\Psi}_{\beta}\left(t, \mathrm{r}^{\prime}\right)\right\}=i \gamma^{0}{ }_{\alpha \beta} \delta^{3}\left(\mathrm{r}-\mathrm{r}^{\prime}\right) \\
& \left\{\hat{\Psi}_{\alpha}(t, \mathrm{r}), \hat{\Psi}_{\alpha}\left(t, \mathrm{r}^{\prime}\right)\right\}=\left\{\hat{\bar{\Psi}}_{\alpha}(t, \mathrm{r}),{ }^{*} \hat{\bar{\Psi}}_{\beta}\left(t, \mathrm{r}^{\prime}\right)\right\}=i \theta_{\alpha \beta} \delta^{3}\left(\mathrm{r}-\mathrm{r}^{\prime}\right)
\end{aligned}
$$

Moreover, the noncommutative fermions propagator $S_{F}\left(x-x^{\prime}\right)$ can be expressed as:

$$
S_{F}\left(x-x^{\prime}\right)=-i\left\langle T\left(\Psi(x) * \bar{\Psi}\left(x^{\prime}\right)\right)\right\rangle=\left\{\begin{array}{l}
\left.\Psi(x) * \bar{\Psi}\left(x^{\prime}\right) \text { if } \mathrm{t}\right\rangle \mathrm{t}^{\prime} \\
\left.\bar{\Psi}\left(x^{\prime}\right) * \Psi(x) \text { if } \mathrm{t}^{\prime}\right\rangle \mathrm{t}
\end{array}\right.
$$

where $T$ denotes to the time-ordered product. In particularly, the study of Kratzer-Fues potential has now become a very interest field due to their applications in different fields [16]. The main motivation of this work is to study and obtaining an analytic expression for the eigenenergies of a (m.k.f.) potential in (NC: 3D-RS) using the Bopp's shift method to discover the new symmetries and a possibility to obtain another applications to this potential in different fields. This work based essentially on our previously works [19-43]. This work is organized as follows: In section 2, we briefly review the Dirac equation with Kratzer-Fues potential on based to Refs. [14-16]. The Section 3 , devoted to studying the three deformed Dirac equation by applying Bopp's shift method. In the fourth section, by applying standard perturbation theory we find the quantum spectrum of the $n^{\text {th }}$ excited states in (NC-3D: RS) symmetries for relativistic spin-orbital interaction with (m.k.f.) potential and then, we derive the corresponding magnetic spectrum. In the section 5, we resume the global spectrum and corresponding noncommutative Hamiltonian operator for (m.k.f.) potential. Finally, in section 6 we present our conclusions.

\section{Review of Dirac equation for Kratzer-Fues potential in ordinary commutative spaces}

We briefly review the differential Dirac equation of a nucleon with mass $M$ moving in both two potentials: attractive scalar potential $S(r)$ and a repulsive vector potential $V(r)[16]$ :

$$
(\alpha \mathrm{P}+\beta(M+S(r))) \Psi(r, \theta, \varphi)=(E-V(r)) \Psi(r, \theta, \varphi)
$$

The Kratzer-Fues potential is given in the slightly modified form:

$$
V(r)=V_{0}\left(1-\frac{r_{0}}{r}\right)^{2}
$$

where $r_{0}$ and $V_{0}$ are the equilibrium separation and is the dissociation energy between diatomic molecules, respectively, which can be simply rewritten in the form 


$$
V(r)=\frac{a}{r^{2}}-\frac{b}{r}+c
$$

with $a=V_{0}{ }^{2} r_{0}{ }^{2}, \quad b=2 V_{0} r_{0}$ and $c=V_{0}$ while $E, \quad M$ and $\left(\alpha_{i}=\left(\begin{array}{cc}0 & \sigma_{i} \\ \sigma_{i} & 0\end{array}\right), \beta=\left(\begin{array}{cc}I_{2 \times 2} & 0 \\ 0 & I_{2 \times 2}\end{array}\right)\right)$ are the fermions' mass, the relativistic energy and the usual Dirac matrices while $\sigma_{1}=\left(\begin{array}{ll}0 & 1 \\ 1 & 0\end{array}\right)$, $\sigma_{2}=\left(\begin{array}{cc}0 & -i \\ i & 0\end{array}\right)$ and $\sigma_{3}=\left(\begin{array}{cc}1 & 0 \\ 0 & -1\end{array}\right)$ are $2 \times 2$ three Pauli matrices. The spinor $\Psi(r, \theta, \varphi)$ determined from the following relation:

$$
\Psi_{n k}(r, \theta, \varphi)=\left(\begin{array}{l}
f_{n k}(\vec{r}) \\
g_{n k}(\vec{r})
\end{array}\right)=\frac{1}{r}\left(\begin{array}{l}
F_{n k}(r) Y_{j m}^{l}(\theta, \varphi) \\
i G_{n \widetilde{k}}(r) Y_{j m}^{\tilde{l}}(\theta, \varphi)
\end{array}\right)
$$

here $k(\tilde{k})$ is related to the total angular momentum quantum numbers for spin symmetry $l$ and pspin symmetry $\tilde{l}$ as [14-16]:

$$
k=\left\{\begin{array}{l}
-(l+1) \text { if }-(\mathrm{j}+1 / 2),\left(\mathrm{s}_{1 / 2}, p_{3 / 2}, \text { etc }\right), j=l+\frac{1}{2}, \text { aligned } \operatorname{spin}(\mathrm{k}\langle 0) \\
+l \quad \text { if } j=l+\frac{1}{2},\left(\mathrm{p}_{1 / 2}, d_{3 / 2}, \text { etc }\right), j=l-\frac{1}{2}, \text { unaligned } \operatorname{spin}(\mathrm{k}>0)
\end{array}\right.
$$

and

$$
\widetilde{k}=\left\{\begin{array}{l}
-\tilde{l} \quad \text { if }-(\mathrm{j}+1 / 2),\left(\mathrm{s}_{1 / 2}, p_{3 / 2}, \text { etc }\right), j=\tilde{l}-\frac{1}{2}, \text { aligned } \operatorname{spin}(\mathrm{k}\langle 0) \\
+(\tilde{l}+1) \text { if } j=\tilde{l}+\frac{1}{2},\left(\mathrm{p}_{1 / 2}, d_{3 / 2}, \text { etc }\right), j=\tilde{l}+\frac{1}{2}, \text { unaligned } \operatorname{spin}(\mathrm{k}>0)
\end{array}\right.
$$

The two radial functions $\left(F_{n k}(r), G_{n k}(r)\right)$ are the upper and lower components of the Dirac spinor which obtained by solving the following differential equations $[14,15,16]$ :

$$
\begin{gathered}
{\left[\frac{d^{2}}{d r^{2}}-\frac{k(k+1)}{r^{2}}-\left(M+E_{n k}-\Delta(r)\left(M-E_{n k}+\Sigma(r)\right)+\frac{\frac{d \Delta(r)}{d r}\left(\frac{d}{d r}+\frac{k}{r}\right)}{M-E_{n k}+\Sigma(r)}\right)\right] F_{n k}(r)=0} \\
{\left[\frac{d^{2}}{d r^{2}}-\frac{k(k-1)}{r^{2}}\left(M+E_{n k}-\Delta(r)\left(M-E_{n k}+\Sigma(r)\right)+\frac{\left.\frac{d \Sigma(r)}{d r}\left(\frac{d}{d r}+\frac{k}{r}\right)\right)}{M+E_{n k}-\Delta(r)}\right) G_{n \tilde{k}}(r)=0\right.}
\end{gathered}
$$

According to The Laplace transform approach (LTA), which applied in refs. $[14,15,16]$, for bound state solutions of the Kratzer-Fues potential for the spin symmetric case, the upper spinor component of the Dirac equation $F_{n k}(r)$ can be calculated as,

$$
F_{n k}(r)=N r^{v+1 / 2} e_{1}^{-\lambda r} F_{1}(-n, 2 v+1,2 \lambda r)
$$

where $N$ and ${ }_{1} F_{1}(-n, 2 v+1,2 \lambda r)$ the normalization constant and the confluent hyper-geometric functions, respectively. Furthermore, the energy eigenvalues of the Dirac equation with KratzerFues potential for the spin symmetry condition is given by [16]:

$$
2 r_{0} V_{0} E_{n k}=\frac{1}{4}+(1+2 n+2) \sqrt{\left.k(k+1)+r_{0}^{2} V_{0} E_{n k}\right)} \sqrt{E_{n k}\left(2 M+V_{0}-C-E_{n k}\right)}
$$


with $n=0,1,2 \ldots$ and the other component $G_{n k}(r)$ can be simply found via $[14,15,16]$ :

$$
G_{n k}(r)=\tilde{N} r^{v+1 / 2} e^{-\lambda r} F_{1}(-n, 2 v+1,2 \lambda r)
$$

where $\tilde{N}$ denote to the normalization constant and energy eigenvalues of the Dirac equation with Kratzer-Fues potential for the pseudo spin symmetry condition is given by[16]:

$$
-2 r_{0} V_{0} \widetilde{E}_{n k}=(1+2 n+2) \sqrt{\left.\tilde{l}(\widetilde{l}+1)-r_{0}^{2} V_{0} \widetilde{E}_{n k}\right)} \sqrt{\widetilde{E}_{n k}\left(2 M-V_{0}+C_{p s}-\widetilde{E}_{n k}\right)}
$$

with $\tilde{l}(\tilde{l}+1)=k(k-1)$ and $\widetilde{E}_{n k}=M-E+C_{p s}$. On the other hand, the generalized Laguerre polynomials $L_{n}^{(\beta)}(r)$ can be expressed as a function of the confluent hyper-geometric functions as [53-55]:

$$
L_{n}^{(p)}(x)=\frac{\Gamma(n+p+1)}{n ! \Gamma(p+1)}{ }_{1} F_{1}(-n, p+1 ; x)
$$

this leads to rewritten Eqs. (24) and (26) as follows, respectively:

$$
F_{n k}(r)=\frac{N n ! \Gamma(2 v+1)}{\Gamma(n+2 v+1)} r^{v+1 / 2} e^{-\lambda r} L_{n}^{(2 v)}(2 \lambda r)
$$

and

$$
G_{n k}(r)=\frac{\tilde{N} n ! \Gamma(2 v+1)}{\Gamma(n+2 v+1)} r^{v+1 / 2} e^{-\lambda r} L_{n}^{(2 v)}(2 \lambda r)
$$

\section{Theory of Noncommutative relativistic Hamiltonian operator for (m.k.f.) potential in (NC-} 3D: RS) symmetries:

\subsection{Formalism of Bopp's shift Method}

Now, we shall review some fundamental principles of the quantum noncommutative relativistic Dirac equation for (m.k.f.) potential $V(\hat{r})$ [19-48]:

- Ordinary Hamiltonian $\hat{H}\left(p_{i}, x_{i}\right)$ will be replace by new noncommutative Hamiltonian $\hat{H}_{n c-k f}\left(\hat{p}_{i}, \hat{x}_{i}\right)$,

- Ordinary spinor $\Psi(\vec{r})$ will be replace by new spinor $\Psi(\vec{r})$,

- Ordinary energy $\mathrm{E}_{\mathrm{nk}}$ replaces by new energy $E_{n c-k f}$ and ordinary product replace by new star product* .

Naively, to get a physical quantity on a noncommutative space, we simply take this quantity on the corresponding commutative space and replace all products by the star products [44], thus we can write the noncommutative relativistic Dirac equation for (m.k.f.) potential as follows:

$$
\hat{H}_{n c-k f}\left(\hat{p}_{i}, \hat{x}_{i}\right) * \hat{\Psi}(\overrightarrow{\vec{r}})=E_{n c-k f} \Psi(\overrightarrow{\vec{r}})
$$

The Bopp's shift method permutes to reduce the above equation using old product with simultaneously translations applied to the operators $\hat{x}_{i}$ and $\hat{p}_{i}$ as follows:

$$
H_{n c-k f}\left(\hat{p}_{i}, \hat{x}_{i}\right) \psi(\vec{r})=E_{n c-k f} \psi(\vec{r})
$$

where the new operator of Hamiltonian $H_{n c-k f}\left(\hat{p}_{i}, \hat{x}_{i}\right)$ can be expressed in three general varieties: both noncommutative space and noncommutative phase (NC-3D: RSP), only noncommutative space (NC-3D: RS) and only noncommutative phase (NC: 3D-RP) as, respectively:

$$
H_{n c-k f}\left(\hat{p}_{i}, \hat{x}_{i}\right) \equiv H\left(\hat{p}_{i}=p_{i}-\frac{1}{2} \bar{\theta}_{i j} x_{j} ; \hat{x}_{i}=x_{i}-\frac{1}{2} \theta_{i j} p_{j}\right) \text { for } \mathrm{NC}-3 \mathrm{D}: \mathrm{RSP}
$$




$$
\begin{aligned}
& H_{n c-k f}\left(\hat{p}_{i}, \hat{x}_{i}\right) \equiv H\left(\hat{p}_{i}=p_{i} ; \hat{x}_{i}=x_{i}-\frac{1}{2} \theta_{i j} p_{j}\right) \text { for NC-3D : RS } \\
& H_{n c-k f}\left(\hat{p}_{i}, \hat{x}_{i}\right) \equiv H\left(\hat{p}_{i}=p_{i}-\frac{1}{2} \bar{\theta}_{i j} ; x_{j}, \hat{x}_{i}=x_{i}\right) \text { for } \mathrm{NC}-3 \mathrm{D}: \mathrm{RP}
\end{aligned}
$$

In this paper we consider the case of only space-space non-commutativity, thus we are interest with the second variety (34) and then the new modified Hamiltonian $H_{n c-k f}\left(\hat{p}_{i}, \hat{x}_{i}\right)$ defined as a function of $\hat{x}_{i}=x_{i}-\frac{1}{2} \theta_{i j} p_{j}$ and $\hat{p}_{i}=p_{i}$ :

$$
H_{n c-k f}\left(\hat{p}_{i}, \hat{x}_{i}\right)=\alpha \hat{\mathrm{P}}+\beta(M+S(\hat{r}))+V(\hat{r})
$$

where the (m.k.f.) potential $V(\hat{r})$ is given by:

$$
V(\hat{r})=\frac{a}{\hat{r}^{2}}-\frac{b}{\hat{r}}+c
$$

In the light of the above considerations, it could be interesting to solve exactly modified Dirac equation for the (m.k.f.) potential $V(\hat{r})$ using Bopp's shift method as follows:

$$
(\alpha \mathrm{P}+\beta(M+S(\hat{r}))) \Psi(r, \theta, \varphi)=(E-V(\hat{r})) \Psi(r, \theta, \varphi)
$$

The radial functions $\left(F_{n k}(r), G_{n k}(r)\right)$ are obtained, by solving two equations:

$$
\begin{aligned}
& {\left[\frac{d}{d r}+\frac{k}{r}\right] F_{n k}(r)=\left[M+E_{n c-m t}-\Delta(\hat{r})\right] G_{n k}(r)} \\
& {\left[\frac{d}{d r}+\frac{k}{r}\right] G_{n k}(r)=\left[M-E_{n c-m t}+\Sigma(\hat{r})\right] G_{n k}(r)}
\end{aligned}
$$

with $\Delta(\hat{r})=V(\hat{r})-S(\hat{r})$ and $\Sigma(\hat{r})=V(\hat{r})+S(\hat{r})$, eliminating $F_{n k}(r)$ and $G_{n k}(r)$ from Eqs. (39) and (40), we can obtain the following two Schrödinger-like differential equations as follows in (NC-3D: RS):

$$
\begin{gathered}
{\left[\frac{d^{2}}{d r^{2}}-\frac{k(k+1)}{r^{2}}-\left(M+E_{n c-k f}-\Delta(\hat{r})\right)\left(M-E_{n c-k f}+\Sigma(\hat{r})\right)\right] F_{n k}(r)=0} \\
{\left[\frac{d^{2}}{d r^{2}}-\frac{k(k-1)}{r^{2}}-\left(M+E_{n c-k f}-\Delta(\hat{r})\right)\left(M-E_{n c-k f}+\Sigma(\hat{r})\right)\right] G_{n k}(r)=0}
\end{gathered}
$$

On based to results of eq. (11), we can easily obtain the two terms $\left(\frac{a}{\hat{r}^{2}}\right)$ and $\left(-\frac{b}{\hat{r}}\right)$, in (NC-3D: RS) spaces as follows:

$$
\begin{array}{r}
\frac{a}{\hat{r}^{2}}=\frac{a}{r^{2}}+\frac{a}{r^{4}} \overrightarrow{\mathbf{L}} \vec{\Theta}+O\left(\theta^{2}\right) \\
-\frac{b}{\hat{r}}=\frac{b}{r}-\frac{b}{2 r^{3}} \overrightarrow{\mathbf{L}} \vec{\Theta}+O\left(\theta^{2}\right)
\end{array}
$$

Substituting (43) into equation (37), we obtain the new potential as:

$$
V(\hat{r})=\frac{a}{r^{2}}-\frac{b}{r}+c+\hat{V}_{p e r t-k f}(r, \Theta, a, b)
$$


with

$$
\hat{V}_{p e r t-k f}(r, \Theta, a, b)=\left(\frac{a}{r^{4}}-\frac{b}{2 r^{3}}\right) \overrightarrow{\mathbf{L}} \vec{\Theta} .
$$

We generalized the constraint for the pseudospin (p-spin) symmetry $(\Delta(r)=V(r)$ and $\Sigma(r)=C_{p s}=$ constants which presented in refs. [14-16] into the new forms $\Delta(\hat{r})=V(\hat{r})$ and $\Sigma(\hat{r})=\hat{C}_{p s}=$ constants in (NC-3D: RS) and inserting the potential $V(\hat{r})$ in eq. (44) into the two Schrödinger-like differential equations (41) and (42), one obtains:

$$
\begin{aligned}
& {\left[\frac{d^{2}}{d r^{2}}-\frac{k(k+1)}{r^{2}}-\left(M+E_{n c-k f}\right)\left(M-E_{n k}+C_{p s}\right)-\left(\frac{a}{r^{2}}-\frac{b}{r}+c\right)\left(M-E_{n k}+C_{p s}\right)-\left(\frac{a}{r^{4}}-\frac{b}{2 r^{3}}\right) \overrightarrow{\mathbf{L}} \vec{\Theta}\left(M-E_{n k}+\hat{C}_{p s}\right)\right] F_{n k}(r)=0} \\
& {\left[\frac{d^{2}}{d r^{2}}-\frac{k(k-1)}{r^{2}}-\left(M+E_{n c-k f}\right)\left(M-E_{n k}+C_{p s}\right)-\left(\frac{a}{r^{2}}-\frac{b}{r}+c\right)\left(M-E_{n k}+C_{p s}\right)-\left(\frac{a}{r^{4}}-\frac{b}{2 r^{3}}\right) \overrightarrow{\mathbf{L}} \vec{\Theta}\left(M-E_{n k}+\hat{C}_{p s}\right)\right] G_{n k}(r)=0}
\end{aligned}
$$

It's clearly that, the additive new part $V_{\text {pert-kf }}(r, \Theta)$ is proportional with infinitesimal parameter $\Theta$, thus we can considered as a perturbations terms.

4. The exact relativistic (p-spin-orbit) and (spin-orbit) Hamiltonian operators and corresponding spectrums for (m.k.f.) potential in (NC: 3D- RS) symmetries:

4.1. The exact relativistic spin-orbital Hamiltonian for (m.k.f.) potential in (NC: 3D- RS) symmetries for one-electron atoms:

In this sub-section, we are going to rewriting the perturbative terms $\hat{V}_{p e r t-k f}(r, \Theta, a, b)$ to the equivalent physical form as follows:

$$
\hat{V}_{p e r t-k f}(r, \Theta, a, b)=\left(\frac{a}{r^{4}}-\frac{b}{2 r^{3}}\right) \stackrel{\vec{S}}{S}
$$

Furthermore, the above perturbative terms $\hat{V}_{p e r t-k f}(r, \Theta, a, b)$ can be rewrite to the following new equivalent expression:

$$
\hat{V}_{\text {pert-mt }}(r, \Theta, a, b)=\frac{1}{2} \Theta\left(\frac{a}{r^{4}}-\frac{b}{2 r^{3}}\right)\left(\vec{J}^{2}-\vec{L}^{2}-\overrightarrow{\widetilde{S}}^{2}\right)
$$

As we know, we just replaced the coupling pseudo spin-orbital (exact spin-orbital) $\overleftrightarrow{\widetilde{S}} \vec{L}$ by the expression $\frac{1}{2}\left(\vec{J}^{2}-\vec{L}^{2}-\overrightarrow{\widetilde{S}}^{2}\right)$, in relativistic quantum mechanics. The set $\left(H_{n c-m t}\left(\hat{p}_{i}, \hat{x}_{i}\right), \mathrm{J}^{2}, \mathrm{~L}^{2}, \widetilde{\mathrm{S}}^{2}\right.$ and $J_{z}$ ) forms a complete of conserved physics quantities and the spin-orbit (pseudo spin-orbit) quantum number $k(\tilde{k})$ is related to the quantum numbers for spin symmetry $l$ and p-spin symmetry $\tilde{l}$ as follows [14-16]:

$$
k=\left\{\begin{array}{l}
k_{1} \equiv-(l+1) \quad \text { if }-(\mathrm{j}+1 / 2),\left(\mathrm{s}_{1 / 2}, p_{3 / 2}, \text { etc }\right), j=l+\frac{1}{2}, \text { aligned } \operatorname{spin}(\mathrm{k}\langle 0) \\
\left.k_{2} \equiv+l \quad \text { if }\left(j=l+\frac{1}{2}\right),\left(\mathrm{p}_{1 / 2}, d_{3 / 2}, \text { etc }\right), j=l-\frac{1}{2}, \text { unaligned } \operatorname{spin}(\mathrm{k}\rangle 0\right)
\end{array}\right.
$$

and 


$$
\tilde{k}=\left\{\begin{array}{l}
\widetilde{k}_{1} \equiv-\tilde{l} \quad \text { if }-(\mathrm{j}+1 / 2),\left(\mathrm{s}_{1 / 2}, p_{3 / 2}, \text { etc }\right), j=\widetilde{l}-\frac{1}{2}, \text { aligned } \operatorname{spin}(\mathrm{k}\langle 0) \\
\left.\widetilde{k}_{2} \equiv+(\tilde{l}+1) \text { if }\left(j=\widetilde{l}+\frac{1}{2}\right),\left(\mathrm{p}_{1 / 2}, d_{3 / 2}, \text { etc }\right), j=\widetilde{l}+\frac{1}{2}, \text { unaligned } \operatorname{spin}(\mathrm{k}\rangle 0\right)
\end{array}\right.
$$

with $\tilde{k}(\tilde{k}-1)=\tilde{l}(\tilde{l}+1)$ and $k(k-1)=l(l+1)$, which allows us to form two diagonal $(3 \times 3)$ matrixes $\hat{H}_{s o-k f}\left(k_{1}, k_{2}\right)$ and $\hat{\widetilde{H}}_{s o-k f}\left(\widetilde{k}_{1}, \widetilde{k}_{2}\right)$, for (m.k.f.) potential, respectively, in (NC: 3D-RS) as:

$\left(\hat{H}_{s o-k f}\right)_{11}\left(k_{1}\right)=k_{1} \Theta\left(\frac{a}{r^{4}}-\frac{b}{2 r^{3}}\right)$ if $-(\mathrm{j}+1 / 2),\left(\mathrm{s}_{1 / 2}, p_{3 / 2}, e t c\right), j=l+\frac{1}{2}$, aligned $\operatorname{spin}(\mathrm{k}\langle 0)$

$\left(\hat{H}_{s o-k f}\right)_{22}\left(k_{2}\right)=k_{2} \Theta\left(\frac{a}{r^{4}}-\frac{b}{2 r^{3}}\right)$ if $\left(j=l+\frac{1}{2}\right),\left(\mathrm{p}_{1 / 2}, d_{3 / 2}, e t c\right), j=l-\frac{1}{2}$, unaligned spin $(\mathrm{k}>0)$

$\left(\hat{H}_{s o-k f}\right)_{33}=0$

and

$$
\begin{aligned}
& \left(\hat{\tilde{H}}_{s o-k f}\right)_{11}\left(\widetilde{k}_{1}\right)=\widetilde{k}_{1} \Theta\left(\frac{a}{r^{4}}-\frac{b}{2 r^{3}}\right) \text { if }-(\mathrm{j}+1 / 2),\left(\mathrm{s}_{1 / 2}, p_{3 / 2}, e t c\right), j=\widetilde{l}-\frac{1}{2}, \text { aligned } \operatorname{spin}(\mathrm{k}\langle 0) \\
& \left(\hat{\tilde{H}}_{s o-k f}\right)_{22}\left(\widetilde{k}_{2}\right)=\widetilde{k}_{2} \Theta\left(\frac{a}{r^{4}}-\frac{b}{2 r^{3}}\right) \text { if }\left(j=\tilde{l}+\frac{1}{2}\right),\left(\mathrm{p}_{1 / 2}, d_{3 / 2}, e t c\right), j=\tilde{l}+\frac{1}{2}, \text { unaligned spin }(\mathrm{k}>0) \\
& \left(\hat{\tilde{H}}_{s o-k f}\right)_{33}=0
\end{aligned}
$$

4.2. The exact relativistic spin-orbital spectrum for (m.k.f.) potential symmetries for $n^{\text {th }}$ excited states for one-electron atoms in (NC: 3D- RSP) symmetries:

In our theoretical work, we are going to study the modifications to the energy levels $\left(E_{n c-p e r \cdot d}\left(\Theta, \tilde{k}_{1}\right), E_{n c-p e r: u}\left(\Theta, \tilde{k}_{2}\right)\right)$ for $\left(-(\mathrm{j}+1 / 2),\left(\mathrm{s}_{1 / 2}, p_{3 / 2}, e t c\right), j=\widetilde{l}+\frac{1}{2}\right.$, aligned spin $\mathrm{k}\langle 0$ and spin-down) and $\left(j=\tilde{l}+\frac{1}{2},\left(\mathrm{p}_{1 / 2}, d_{3 / 2}\right.\right.$, etc $), j=\tilde{l}-\frac{1}{2}$, un aligned spin $\mathrm{k}>0$ and spin up), respectively, at first order of infinitesimal parameter $\Theta$, for $n^{\text {th }}$ excited states, obtained by applying the standard perturbation theory, using equations (30), (48) and (51) as:

$$
\begin{aligned}
& E_{n c-p e r \cdot d}\left(\Theta, \widetilde{k}_{1}\right) \equiv \Theta \widetilde{k}_{1}\left(\frac{\tilde{N} n ! \Gamma(2 v+1)}{\Gamma(n+2 v+1)}\right)^{2} \int r^{2 v+1} e^{-2 \lambda r}\left[L_{n}^{(2 v)}(2 \lambda r)\right]^{2}\left(\frac{a}{r^{4}}-\frac{b}{2 r^{3}}\right) r^{2} d r \\
& E_{n c-p e r: u}\left(\Theta, \widetilde{k}_{2}\right) \equiv \Theta \widetilde{k}_{2}\left(\frac{\tilde{N} n ! \Gamma(2 v+1)}{\Gamma(n+2 v+1)}\right)^{2} \int r^{2 v+1} e^{-2 \lambda r}\left[L_{n}^{(2 v)}(2 \lambda r)\right]^{2}\left(\frac{a}{r^{4}}-\frac{b}{2 r^{3}}\right) r^{2} d r
\end{aligned}
$$

A direct simplification gives:

$$
\begin{gathered}
E_{n c-p e r: d}\left(\Theta, \widetilde{k}_{1}\right) \equiv \Theta \widetilde{k}_{1}\left(\frac{\tilde{N} n ! \Gamma(2 v+1)}{\Gamma(n+2 v+1)}\right)^{2}\left(T_{1-k f}+T_{2-k f}\right) \\
E_{n c-p e r: u}\left(\Theta, \widetilde{k}_{2}\right) \equiv \Theta \widetilde{k}_{2}\left(\frac{\widetilde{N} n ! \Gamma(2 v+1)}{\Gamma(n+2 v+1)}\right)^{2}\left(T_{1-k f}+T_{2-k f}\right)
\end{gathered}
$$


where, the two terms $T_{1-k f}$ and $T_{2-k f}$ are given by:

$$
\begin{aligned}
& T_{1-k f}=a \int_{0}^{+\infty} r^{2 v-3} e^{-2 \lambda r}\left[L_{n}^{(2 v)}(2 \lambda r)\right]^{2} d r \\
& T_{2-k f}=-\frac{b}{2} \int_{0}^{+\infty} r^{2 v-2} e^{-2 \lambda r}\left[L_{n}^{(2 v)}(2 \lambda r)\right]^{2} d r
\end{aligned}
$$

Defining the new variable $2 \lambda r \equiv X$ and rewriting the two factors $T_{1-k f}$ and $T_{2-k f}$ as:

$$
\begin{aligned}
& T_{1-k f}=\frac{a}{(2 \lambda)^{2 v-3}} \int_{0}^{+\infty} X^{2 v-3} e^{-X}\left[L_{n}^{(2 v)}(X)\right]^{2} d X \\
& T_{2-k f}=-\frac{b}{2(2 \lambda)^{2 v-2}} \int_{0}^{+\infty} X^{2 v-2} e^{-X}\left[L_{n}^{(2 v)}(X)\right]^{2} d X
\end{aligned}
$$

In order to obtain $T_{1-k f}$ and $T_{2-k f}$, we apply the following special integral [60,61]:

$$
J_{n, \alpha}^{(\gamma)}=\int_{0}^{\infty} e^{-x} x^{\alpha+\gamma}\left[L_{n}^{\alpha}(x)\right]^{2} d x=\frac{(\alpha+n) !}{n !} \sum_{i=0}^{n}(-1)^{i} \frac{\Gamma(n+i+\gamma)}{\Gamma(-i-\gamma)} \frac{(\alpha+i+\gamma) !}{(\alpha+i) !} \frac{1}{i !(n-i) !}
$$

With $\operatorname{Re}(\alpha+\gamma+1)\rangle 0, \gamma$ can be takes: $(-3,-2)$ and $\alpha \rightarrow 2 v$, which allow us to obtaining $T_{1-k f}$ and $T_{2-k f}$ as:

$$
\begin{aligned}
& T_{1-k f}=\frac{a}{(2 \lambda)^{2 v-3}} \frac{(2 v+n) !}{n !} \sum_{i=0}^{n}(-1)^{i} \frac{\Gamma(n+i-3)}{\Gamma(3-i)} \frac{(2 v+i-3) !}{(2 v+i) !} \frac{1}{i !(n-i) !} \\
& T_{2-k f}=-\frac{b}{2(2 \lambda)^{2 v-2}} \frac{(2 v+n) !}{n !} \sum_{i=0}^{n}(-1)^{i} \frac{\Gamma(n+i-2)}{\Gamma(2-i)} \frac{(2 v+i-2) !}{(2 v+i) !} \frac{1}{i !(n-i) !}
\end{aligned}
$$

Substituting Eqs. (61) and (62) into Eqs. (56) and (57), we obtain the exact modifications of fundamental states $\left(E_{n c-p e r d}\left(\Theta, \widetilde{k}_{1}\right), E_{n c-p e r: u}\left(\Theta, \widetilde{k}_{2}\right)\right)$ produced by relativistic p-spin-orbital effect:

$$
\begin{aligned}
& E_{n c-p e r \cdot d}\left(\Theta, \widetilde{k_{1}}\right) \equiv \Theta \tilde{k_{1}} n !\left(\frac{\tilde{N} \Gamma(2 v+1)}{\Gamma(n+2 v+1)}\right)^{2}(2 v+n) ! \\
& \cdot\left(\frac{a}{(2 \lambda)^{2 v-3}} \sum_{i=0}^{n}(-1) \frac{\Gamma(n+i-3)}{\Gamma(3-i)} \frac{(2 v+i-3) !}{(2 v+i) !} \frac{1}{i !(n-i) !}-\frac{b}{2(2 \lambda)^{2 v-2}} \sum_{i=0}^{n}(-1)^{i} \frac{\Gamma(n+i-2)}{\Gamma(2-i)} \frac{(2 v+i-2) !}{(2 v+i) !} \frac{1}{i !(n-i) !}\right) \\
& E_{n c-p e r: u}\left(\Theta, \widetilde{k}_{2}\right) \equiv \Theta \tilde{k}_{2} n !\left(\frac{\tilde{N} \Gamma(2 v+1)}{\Gamma(n+2 v+1)}\right)^{2}(2 v+n) ! \\
& \cdot\left(\frac{a}{(2 \lambda)^{2 v-3}} \sum_{i=0}^{n}(-1)^{i} \frac{\Gamma(n+i-3)}{\Gamma(3-i)} \frac{(2 v+i-3) !}{(2 v+i) !} \frac{1}{i !(n-i) !}-\frac{b}{2(2 \lambda)^{2 v-2}} \sum_{i=0}^{n}(-1)^{i} \frac{\Gamma(n+i-2)}{\Gamma(2-i)} \frac{(2 v+i-2) !}{(2 v+i) !} \frac{1}{i !(n-i) !}\right)
\end{aligned}
$$

4.3. The exact relativistic magnetic spectrum for (m.k.f.) potential for $n^{\text {th }}$ excited states for one-electron atoms in (NC: 3D- RS) symmetries:

Having obtained the exact modifications to the energy levels $\left(E_{n c-p e r d}\left(\Theta, \widetilde{k}_{1}\right), E_{n c-p e r: u}\left(\Theta, \widetilde{k}_{2}\right)\right)$, for $n^{\text {th }}$ exited states, produced with relativistic spin-orbital induced by noncommutative p-spinorbital Hamiltonian operator, we now turn our attention to the study another interested physically 
meaningful phenomena, which also produced from the perturbative terms of modified Kratzer-Fues potential related to the influence of an external uniform magnetic field, it's sufficient to apply the following two replacements to describing these phenomena:

$$
\left(\frac{a}{r^{4}}-\frac{b}{2 r^{3}}\right) \overrightarrow{\mathbf{L}} \vec{\Theta} \rightarrow \chi\left(\frac{a}{r^{4}}-\frac{b}{2 r^{3}}\right) \vec{B} \vec{L} \text { and } \Theta \rightarrow \chi B
$$

Here $\chi$ is infinitesimal real proportional's constants, and we choose the magnetic field $\overleftrightarrow{B}=B \vec{k}$, which allow us to introduce the modified new magnetic Hamiltonian $\hat{H}_{m a g-k f}(r, a, b, \chi)$ in (NC: 3DRS), as:

$$
\hat{H}_{\text {mag-kf }}(r, a, b, \chi)=\chi\left(\frac{a}{r^{4}}-\frac{b}{2 r^{3}}\right)(\vec{B} \vec{J}-\stackrel{\vec{S}}{\leftrightarrow} \vec{B})
$$

here $(-\overleftrightarrow{S} \overleftrightarrow{B})$ denote to the ordinary Hamiltonian of Zeeman Effect. To obtain the exact noncommutative magnetic modifications of energy $E_{\text {magkf }}(\chi, n, \widetilde{m}, a, b)$ for modified Kratzer-Fues potential, which produced automatically by the effect of $\hat{H}_{m-k f}(r, a, b, \chi)$, we make the following two simultaneously replacements:

$$
\widetilde{k}_{1} \rightarrow \tilde{\mathrm{m}} \text { and } \Theta \rightarrow \chi
$$

Thus, we obtain the magnetic energy spectrum $E_{\text {magkf }}(\chi, n, \tilde{m}, a, b)$, corresponding $n^{\text {th }}$ excited states, for the (m.k.f.) potential in view of pseudospin symmetric in (NC-3D: RS) symmetries as,

$$
\begin{aligned}
& E_{\mathrm{mag}-\mathrm{mt}}(\chi, n, \tilde{m}, a, b)=\chi \tilde{m} B n !\left(\frac{\tilde{N} \Gamma(2 v+1)}{\Gamma(n+2 v+1)}\right)^{2}(2 v+n) ! \\
& \cdot\left(\frac{a}{(2 \lambda)^{2 v-3}} \sum_{i=0}^{n}(-1)^{i} \frac{\Gamma(n+i-3)}{\Gamma(3-i)} \frac{(2 v+i-3) !}{(2 v+i) !} \frac{1}{i !(n-i) !}-\frac{b}{2(2 \lambda)^{2 v-2}} \sum_{i=0}^{n}(-1)^{i} \frac{\Gamma(n+i-2)}{\Gamma(2-i)} \frac{(2 v+i-2) !}{(2 v+i) !} \frac{1}{i !(n-i) !}\right)
\end{aligned}
$$

where $\tilde{m}$ denote to the angular momentum quantum number, $-\tilde{l} \leq \tilde{m} \leq+\tilde{l}$, which allow us to fixing $(2 \tilde{l}+1)$ values for the orbital angular momentum quantum numbers, thus we can obtain the second class of solutions for modified Kratzer-Fues potential.

\section{Results}

Following the discussions of the Sect. 4, we proceed now to find the eigenvalues for this problem for $n^{\text {th }}$ excited states $\left(E_{n c-d}\left(\Theta, \widetilde{k}_{1}, \chi, n, \tilde{m}, a, b\right)\right.$ and $\left.E_{n c r u}\left(\Theta, \widetilde{k}_{2}, \chi, n, \widetilde{m}, a, b\right)\right)$ of modified Dirac equation corresponding for $\left(-(\mathrm{j}+1 / 2),\left(\mathrm{s}_{1 / 2}, p_{3 / 2}\right.\right.$, etc $), j=\tilde{l}+\frac{1}{2}$, aligned spin $\mathrm{k}\langle 0$ and spin-down $)$ and $\left(j=\tilde{l}+\frac{1}{2},\left(\mathrm{p}_{1 / 2}, d_{3 / 2}, e t c\right), j=\tilde{l}-\frac{1}{2}\right.$, un aligned spin $\mathrm{k}>0$ and spin up), respectively, at first order of parameter $\Theta$, for (m.k.f.) potential in (NC: 3D-RS) symmetries, on based to the obtained new results (63), (64) and (68), in addition to the original results (27) of energies in commutative space, we obtain the following original results:

$$
\begin{aligned}
& E_{n c-d}\left(\Theta, \widetilde{k}_{1}, \chi, n, \widetilde{m}, a, b\right)=E_{n \widetilde{k_{1}}}+n !\left(\frac{\tilde{N} \Gamma(2 v+1)}{\Gamma(n+2 v+1)}\right)^{2}(2 v+n) ! \\
& \cdot\left(\frac{a}{(2 \lambda)^{2 v-3}} \sum_{i=0}^{n}(-1)^{i} \frac{\Gamma(n+i-3)}{\Gamma(3-i)} \frac{(2 v+i-3) !}{(2 v+i) !} \frac{1}{i !(n-i) !}-\frac{b}{2(2 \lambda)^{2 v-2}} \sum_{i=0}^{n}(-1)^{i} \frac{\Gamma(n+i-2)}{\Gamma(2-i)} \frac{(2 v+i-2) !}{(2 v+i) !} \frac{1}{i !(n-i) !}\right) \\
& \left\{\Theta \widetilde{k_{1}}+\chi \widetilde{m} B\right\}
\end{aligned}
$$




$$
\begin{aligned}
& E_{n c-u}\left(\Theta, \widetilde{k}_{2}, \chi, n, \tilde{m}, a, b\right)=E_{n \widetilde{k}_{2}}+E_{n \widetilde{k}_{1}}+n !\left(\frac{\tilde{N} \Gamma(2 v+1)}{\Gamma(n+2 v+1)}\right)^{2}(2 v+n) ! \\
& \cdot\left(\frac{a}{(2 \lambda)^{2 v-3}} \sum_{i=0}^{n}(-1)^{i} \frac{\Gamma(n+i-3)}{\Gamma(3-i)} \frac{(2 v+i-3) !}{(2 v+i) !} \frac{1}{i !(n-i) !}-\frac{b}{2(2 \lambda)^{2 v-2}} \sum_{i=0}^{n}(-1)^{i} \frac{\Gamma(n+i-2)}{\Gamma(2-i)} \frac{(2 v+i-2) !}{(2 v+i) !} \frac{1}{i !(n-i) !}\right) \\
& \left\{\Theta \widetilde{k}_{2}+\chi \widetilde{m} B\right\}
\end{aligned}
$$

This is exactly the spectrum of three-dimensional Kratzer-Fues potential. As it is montionated in ref. [14], in view of exact spin symmetry in commutative space $\left(E_{n k} \rightarrow-E_{n k}, V(r) \rightarrow-V(r), k \rightarrow k+1\right.$ and $G_{n \widetilde{k}}(r) \rightarrow F_{n k}(r)$ ), we need to generalize the above translations to the case of noncommutative three dimensional spaces as:

$$
\begin{aligned}
& E_{n c-d}\left(\Theta, \widetilde{k}_{1}, \chi, n, \tilde{m}, a, b\right) \rightarrow E_{n c-d}\left(\Theta, k_{1}, \chi, n, m, a, b\right) \equiv-E_{n c-d}\left(\Theta, \widetilde{k}_{1}+1, \chi, n, m, a, b\right) \\
& E_{n c-u}\left(\Theta, \widetilde{k}_{1}, \chi, n, \tilde{m}, a, b\right) \rightarrow E_{n c-u}\left(\Theta, k_{1}, \chi, n, m, a, b\right) \equiv-E_{n c-u}\left(\Theta, \widetilde{k}_{2}+1, \chi, n, m, a, b\right) \\
& V(\hat{r}) \rightarrow-V(\hat{r}) \\
& \widetilde{k}_{1} \rightarrow k_{1}+1 \quad \text { and } \quad \widetilde{k}_{2} \rightarrow k_{2}+1
\end{aligned}
$$

The spin symmetry in the modified Dirac equation occurs when the difference of the potential between the vector potential $V(\hat{r})$ and scalar potential $S(\hat{r})$ is a constant $\Delta(\hat{r})=V(\hat{r})-S(\hat{r})=$ const, thus, we obtain the energy spectrum for the asymmetric trigonometric Kratzer-Fues potential in view of exact spin symmetry. On the other hand, the obtained eigenvalues of energies are real and then the noncommutative Hamiltonian operator $\hat{H}_{n c-k f}$ is Hermitian, it is natural to consider is the sum of ordinary Hamiltonian operator and the parts: (36), (52), (53) and (66) corresponding p-spin-orbit and Zeeman effect, respectively, thus, we can expressed $\hat{H}_{n c-k f}$ as follows:

$$
\begin{aligned}
& \hat{H}_{n c-k f}\left(\hat{p}_{i}, \hat{x}_{i}\right)=\hat{H}_{c o m-k f}\left(p_{i}, x_{i}\right) \\
& +\left\{\begin{array}{l}
\Theta\left(\frac{a}{r^{4}}-\frac{b}{2 r^{3}}\right) \overleftrightarrow{S} \overleftrightarrow{L}+\chi\left(\frac{a}{r^{4}}-\frac{b}{2 r^{3}}\right)(\vec{B} \vec{J}-\overrightarrow{\widetilde{S}} \stackrel{\leftrightarrow}{B}) \text { for the spin symmetric case } \\
\Theta\left(\frac{a}{r^{4}}-\frac{b}{2 r^{3}}\right) \overrightarrow{\widetilde{S}} \overleftrightarrow{L}+\chi\left(\frac{a}{r^{4}}-\frac{b}{2 r^{3}}\right)(\vec{B} \vec{J}-\overrightarrow{\widetilde{S}} \stackrel{\leftrightarrow}{B}) \text { for the p - spin symmetric case }
\end{array}\right.
\end{aligned}
$$

where $\hat{H}_{c o m-k f}\left(p_{i}, x_{i}\right)$ denote to ordinary quantum Hamiltonian operator. In this way, one can obtain the complete energy spectra and corresponding noncommutative Hamiltonian operator for (m.k.f.) potential in (NC: 3D-RS) symmetries. Now the following accompanying constraint relations:

- The original spectrum contains two possible values of energies in ordinary three dimensional spaces which presented by equations (63), (64) and (68),

- The quantum number $\widetilde{m}$ satisfied the interval: $-\tilde{l} \leq \widetilde{m} \leq+\tilde{l}$, thus we have $(2 \tilde{l}+1)$ values for this quantum number,

- We have also two values for $j=\tilde{l}+\frac{1}{2}$ and $j=\tilde{l}-\frac{1}{2}$.

Allow us to deduce the important original results: every state in usually three dimensional space will be replace by $2(2 \tilde{l}+1)$ sub-states and then the degenerated state can be take $2 \sum_{i=0}^{n-1}(2 l+1) \equiv 2 n^{2}$ values in (NC: 3D-RS) symmetries. 


\section{Conclusion}

In this study we have exactly solved the new radial Dirac equations for the modified KratzerFues potential potentials by using Boopp's shift method. We considered the case of only spacespace non-commutativity in three dimensions; we have found the energy eigenvalues and the corresponding Hermitian anisotropic Hamiltonian operator of the one-electron atoms. We observed that our theoretical results are depended on two infinitesimal parameters $(\Theta, \chi)$ and the discreet atomic quantum numbers $(j=\tilde{l} \pm 1 / 1, s= \pm 1 / 2, \tilde{l}$ and $\tilde{m})$. Due to the full and rich results we hope to discover another physical application to this potential in different fields.

\section{Acknowledgements}

This work was supported with search laboratory of: Physics and Material Chemistry, in Physics department, Sciences faculty, University of M'sila, Algeria.

\section{References}

[1] E. Maghsoodi, H. Hassanabadi, O. Aydoğdu, Dirac particles in the presence of the Yukawa potential plus a tensor interaction in SUSYQM framework, Physica Scripta. 86(1) (2012) 015005.

[2] E. Maghsoodi et al., Relativistic symmetries of the Dirac equation under the nuclear WoodsSaxon potential, Physica Scripta. 85(5) (2012) 055007.

[3] M. Hamzavi, S.M. Ikhdair, B.I. Ita, Approximate spin and pseudospin solutions to the Dirac equation for the inversely quadratic Yukawa potential and tensor interaction, Physica Scripta. 85(4) (2012) 045009.

[4] S.M. Ikhdair, B.J. Falaye, Approximate relativistic bound states of a particle in Yukawa field with Coulomb tensor interaction, Physica Scripta. 87(3) (2013) 035002.

[5] S.M. Ikhdair, Approximate $\kappa$-state solutions of the Dirac equation in spatially dependent mass for the Eckart potential including the Yukawa tensor interaction, Physica Scripta. 88(6) (2013) 065007.

[6] S.H. Dong, Z.Q. Ma, Exact solutions to the Dirac equation with a Coulomb potential in $2+1$ dimensions, Phys. Lett. A. 312 (2003) 78-83.

[7] S. Zarrinkamar, H. Hassanabadi, A.A. Rajabi, Dirac equation for a Coulomb scalar, vector and tensor interaction, Int. J. Mod. Phys. A. 26 (2011) 1011-1018.

[8] K.J. Oyewumi et al., k-state solutions for the fermionic massive spin-1/2 particles interacting with double ring-shaped Kratzer and oscillator potentials, Int. J. Mod. Phys. E. 23 (2014) 1450005 .

[9] H. Hassanabadi, E. Maghsoodi, S. Zarrinkamar, Relativistic symmetries of Dirac equation and the Tietz potential, The European Physical Journal Plus. 127(3) (2012) 1-14.

[10] M.R. Setare, S. Haidari, Spin symmetry of the Dirac equation with the Yukawa potential, Physica Scripta. 81 (2010) 065201.

[11] A. Soylu, O. Bayrak, I. Boztosun, An approximate solution of the Dirac-Hulth_n problem with pseudospin and spin symmetry for any k state, J. Math.Phys. 48 (2007) 082302.

[12] C.A. Onate, J.O. Ojonubah, Relativistic and nonrelativistic solutions of the generalized Pöschl- Teller and hyperbolical potentials with some thermodynamic properties, Int. J. Mod. Phys. E. 24(03) (2015) 1550020. 
[13] S.M. Ikhdair, R. Sever, Approximate analytical solutions of the generalized Woods-Saxon potentials including the spin-orbit coupling term and spin symmetry, Cent. Eur. J. Phys. 8(4) (2010) 652-666.

[14] S.M. Ikhdair, R. Sever, Approximate Eigenvalue and Eigenfunctions Solutions for the Generalized Hulthén Potential with any Angular Momentum, Journal of Mathematical Chemistry. 42(3) (2007) 461-471.

[15] M. Eshghia, S.M. Ikhdair, Relativistic effect of pseudospin symmetry and tensor coupling on the Mie-type potential via Laplace transformation method, Chin. Phys. B. 23(12) (2014) 120304.

[16] B. Biswas, S. Debnath, Bound States of the Dirac-Kratzer-Fues Problem with Spin and Pseudo-Spin Symmetry via Laplace Transform Approach, Bulg. J. Phys. 43 (2016) 89-99.

[17] H.S. Snyder, Quantized Space-Time, Phys. Rev. 71(1) (1947) 38-41.

[18] A. Connes, Noncommutative differential geometry, Publications mathématiques de l'IHÉS, 62(1) (1985) 41-144.

[19] A. Maireche, Atomic Spectrum for Schrödinger Equation with Rational Spherical Type Potential in Non-commutative Space and Phase, Afr. Rev Phys. 10 (2015) 373-381.

[20] A. Maireche, Spectrum of Hydrogen Atom Ground State Counting Quadratic Term in Schrödinger Equation, Afr. Rev Phys. 10 (2015) 177-183.

[21] A. Maireche, Deformed Bound States for Central Fraction Power Potential: Non Relativistic Schrödinger Equation, Afr. Rev Phys. 10 (2015) 97-103.

[22] A. Maireche, Spectrum of Schrödinger Equation with H.L.C. Potential in Non-commutative Two-dimensional Real Space, Afr. Rev Phys. 9 (2014) 479-485.

[23] A. Maireche, A Study of Schrödinger Equation with Inverse Sextic Potential in 2-dimensional Non-commutative Space, Afr. Rev Phys. 9 (2014) 185-193.

[24] A. Maireche, Nonrelativistic Atomic Spectrum for Companied Harmonic Oscillator Potential and its Inverse in both NC-2D: RSP, International Letters of Chemistry, Physics and Astronomy. 56 (2015) 1-9.

[25] A. Maireche, New Exact Solution of the Bound States for the Potential Family V(r) $=\mathrm{A} / \mathrm{r}^{2}$ $\mathrm{B} / \mathrm{r}+\mathrm{Cr}^{\mathrm{k}}(\mathrm{k}=0,-1,-2)$ in both Noncommutative Three Dimensional Spaces and Phases: Non Relativistic Quantum Mechanics, International Letters of Chemistry, Physics and Astronomy. 58 (2015) 164-176.

[26] A. Maireche, A New Approach to the Non Relativistic Schrödinger Equation for an EnergyDepended Potential $V\left(r, E_{n, l}\right)=V_{0}\left(1+\eta E_{n, l}\right) r^{2}$ in both Noncommutative Three Dimensional Spaces and Phases, International Letters of Chemistry, Physics and Astronomy. 60 (2015) 11-19.

[27] A. Maireche, A New Study to the Schrödinger Equation for Modified Potential V(r) $=\operatorname{ar}^{2}+$ $\mathrm{br}^{-4}+\mathrm{cr}^{-6}$ in Nonrelativistic Three Dimensional Real Spaces and Phases, International Letters of Chemistry, Physics and Astronomy. 61 (2015) 38-48.

[28] A. Maireche, A New Nonrelativistic Investigation for the Lowest Excitations States of Interactions in One-Electron Atoms, Muonic, Hadronic and Rydberg Atoms with Modified Inverse Power Potential, International Frontier Science Letters. 9 (2016) 33-46.

[29] A. Maireche, Deformed Quantum Energy Spectra with Mixed Harmonic Potential for Nonrelativistic Schrödinger equation, J. Nano-Electron. Phys. 7(2) (2015) 02003-1 - 02003-6. 
[30] A. Maireche, A Recent Study of Quantum Atomic Spectrum of the Lowest Excitations for Schrödinger Equation with Typical Rational Spherical Potential at Planck's and Nanoscales, J. Nano- Electron. Phys. 7(3) (2015) 03047-1 - 03047-7

[31] A. Maireche, Quantum Hamiltonian and Spectrum of Schrödinger Equation with companied Harmonic Oscillator Potential and its Inverse in three Dimensional Noncommutative Real Space and Phase, J. Nano- Electron. Phys. 7(4) (2015) 04021-1 - 04021-7.

[32] A. Maireche, New Relativistic Atomic Mass Spectra of Quark ( $u, d$ and $s$ ) for Extended Modified Cornell Potential in Nano and Plank's Scales, J. Nano- Electron. Phys. 8(1) (2016) 01020-1 - 01020-7.

[33] A. Maireche, The Nonrelativistic Ground State Energy Spectra of Potential Counting Coulomb and Quadratic Terms in Non-commutative Two Dimensional Real Spaces and Phases, J. Nano- Electron. Phys. 8(1) (2016) 01021-1 - 01021-6.

[34] A. Maireche, New Theoretical Study of Quantum Atomic Energy Spectra for Lowest Excited States of Central (PIHOIQ) Potential in Noncommutative Spaces and Phases Symmetries at Plan's and Nanoscales, J. Nano- Electron. Phys. 8(2) (2016) 02027-1 - 02027-10.

[35] A. Maireche, A New Nonrelativistic Atomic Energy Spectrum of Energy Dependent Potential for Heavy Quarkouniom in Noncommutative Spaces and Phases Symmetries, J. NanoElectron. Phys. 8(2) (2016) 02046-1 - 02046-6.

[36] A. Maireche, New exact bound states solutions for (C.F.P.S.) potential in the case of Noncommutative three dimensional non relativistic quantum mechanics, Med. J. Model. Simul. 04 (2015) 060-072.

[37] A. Maireche, New Bound State Energies for Spherical Quantum Dots in Presence of a Confining Potential Model at Nano and Plank's Scales, NanoWorld J. 1(4) (2016) 120-127.

[38] A. Maireche, Quantum Schrödinger equation with Octic potential in non-commutative twodimensional complex space, Life Sci J. 11(6) (2014) 353-359.

[39] A. Maireche, New quantum atomic spectrum of Schrödinger equation with pseudo harmonic potential in both noncommutative three dimensional spaces and phases, Lat. Am. J. Phys. Educ. 9(1) (2015) 1301.

[40] A. Maireche, D. Imane, A New Nonrelativistic Investigation for Spectra of Heavy Quarkonia with Modified Cornell Potential: Noncommutative Three Dimensional Space and Phase Space Solutions, J. Nano-Electron. Phys. 8(3) (2016) 03025-1.

[41] A. Maireche, A Complete Analytical Solution of the Mie-Type Potentials in Noncommutative 3-Dimensional Spaces and Phases Symmetries, Afr. Rev Phys. 11 (2016) 111 117.

[42] A. Maireche, New Exact Energy Eigen-values for (MIQYH) and (MIQHM) Central Potentials:Non-relativistic Solutions, Afr. Rev Phys. 11 (2016) 175-185.

[43] A. Maireche, A New Relativistic Study for Interactions in One-electron atoms (Spin 1/2 Particles) with Modified Mie-type Potential, J. Nano-Electron. Phys. 8(4) (2016) 04027-1 04027-9.

[44] T. Curtright, D. Fairlie, C.K. Zachos, Features of time independent Wigner functions, Phys.Rev. D. 58(2) (1998) 025002.

[45] J. Gamboa, M. Loewe, J.C. Rojas, Noncommutative quantum mechanics, Phys. Rev. D. 64(6) (2001) 067901. 
[46] L. Mezincescu, Star operation in quantum mechanics, Unpublished paper, arXiv preprint hepth/0007046v2, 2000.

[47] Y. Yi et al., Spin-1/2 relativistic particle in a magnetic field in NC phase space, Chinese Physics C. 34(5) (2010) 543-547.

[48] A.E.F. Djemei, H. Smail, On Quantum Mechanics on Noncommutative Quantum Phase Space, Communications in Theoretical Physics. 41(6) (2004) 837-844.

[49] S. Cai, T. Jing, Dirac Oscillator in Noncommutative Phase Space, Int. J. Theor. Phys. 49(8) (2010) 1699-1705.

[50] J. Lee, Star Products and the Landau Problem, Journal of the Korean Physical Society. 47(4) (2005) 571-576.

[51] A.F. Dossa, G.Y.H. Avossevou, Noncommutative phase space and the two dimensional quantum dipole in background electric and magnetic fields, Journal of Modern Physics. 4(10) (2013) 1400-1411.

[52] Z.H. Yang et al., DKP Oscillator with Spin-0 in Three-dimensional Noncommutative Phase Space, Int. J. Theor. Phys. 49 (2010) 644-657.

[53] J. Mamat; S. Dulat, H. Mamatabdulla, Landau-like Atomic Problem on a Non-commutative Phase Space, Int. J. Theor. Phys. 55 (2016) 2913-2918.

[54] B. Mirza et al., Relativistic Oscillators in a Noncommutative Space and in a Magnetic Field, Commun. Theor. Phys. 55 (2011) 405-409.

[55] Y. Xiao; Z. Long, S. Cai, Klein-Gordon Oscillator in Noncommutative Phase Space Under a Uniform Magnetic Field, Int. J. Theor. Phys. 50 (2011) 3105-3111.

[56] A. Al-Jamel, Heavy quarkonia with Cornell potential on noncommutative space, Journal of Theoretical and Applied Physics. 5(1) (2011) 21-24.

[57] H. Hassanabadi, F. Hoseini, S. Zarrinkamar, A generalized interaction in noncommutative space: Both relativistic and nonrelativistic fields, Eur. Phys. J. Plus. 130(10) (2015) 1-7.

[58] W.S. Chung, Two Dimensional Non-commutative Space and Rydberg Atom Mode, Int. J. Theor. Phys. 54(6) (2015) 1840-1849.

[59] M.M. Nieto, L.M. Simmons Jr., Eigenstates, Coherent States, and Uncertainty Products for the Morse Oscillator, Phys. Rev. A. 19(2) (1979) 438-444.

[60] M. Abramowitz, I.A. Stegun, Handbook of Mathematical Functions with Formulas, Graphs, and Mathematical Tables, Dover Publications, New York, 1965.

[61] S.H. Dong, G.H. Sun, The Schrödinger Equation with a Coulomb Plus Inverse-Square Potential in D Dimensions, Phys. Scr. 70(2-3) (2004) 94-97. 